\title{
Impacto en las Áreas de Desempeño Ocupacional como Consecuencia de una Fobia Social
}

\author{
Impact of Social Phobia in Occupational Performance’s Areas
}

Laura Rueda C.

Col. Ana María Arias Q. a , Daniela Dinamarca S. b, Daniela Esmar G ${ }^{\mathrm{C}}$. Elba Lizama C. ${ }^{\text {de }}$.

\section{RESUMEN}

Dentro de los trastornos de ansiedad se encuentra la fobia social, cuya sintomatología se caracteriza por: angustia anticipatoria, ansiedad de exposición y evitación, frente a las situaciones sociales.

Considerando las características de la patología, la hipótesis de esta investigación es que la fobia social provoca un impacto en la ocupación de las personas afectadas, y por ende en las áreas de desempeño, lo cual repercute negativamente en la calidad de vida de los individuos.

Los objetivos del trabajo son por un lado determinar cómo se alteran las áreas de desempeño ocupacional en personas con fobia social. Además, se desea también establecer funciones del Terapeuta Ocupacional en el tratamiento de personas con este trastorno.

Se trata de una investigación de tipo cualitativo utilizando una entrevista semi estructurada para la recolección de datos y a través del relato de historia de vida, se determinan ejes de análisis para comprender el impacto de la Fobia Social.

Palabras clave: trastornos de ansiedad, fobia social, desempeño ocupacional.

\section{ABSTRACT}

Social phobia is included on the anxiety disorders group, with symptoms like: advance anguish, exposure anxiety and avoidance, in all social situations.

Considering symptoms of this disorder, research's hypothesis is social phobia affects patient's occupations and therefore performance's areas, wich strikes in a negative way on his or her life's quality.

\footnotetext{
a Terapeuta Ocupacional, Licenciada en Ciencias de la Ocupación Humana. Terapeuta Ocupacional de la Corporación Municipal de Puente Alto, Programa de Bienestar Psicosocial Estudiantil. Cel: 09-9970021. E-mail: negrit@hotmail.com.

${ }^{\mathrm{b}}$ Terapeuta Ocupacional, Licenciada en Ciencias de la Ocupación Humana.. Cel: 08-6022826. E-mail: danieladinamarca@yahoo.com.

${ }^{\mathrm{C}}$ Terapeuta Ocupacional, Licenciada en Ciencias de la Ocupación Humana.. Cel: 08-2638492. E-mail: dani_esmar@yahoo.com.

derapeuta Ocupacional, Licenciada en Ciencias de la Ocupación Humana. Cel: 08-4841803. E-mail: elba.lizama@gmail.com.

e Investigadora responsable, Terapeuta Ocupacional, Licenciada en Filosofía Licenciada en Ciencias de la Ocupación Humana, Magíster en Bioética. Profesora Asistente Escuela de Terapia Ocupacional. Facultad de Medicina Universidad de Chile Lrueda@med.uchile.cl 9876344
} 
The aims of the work are on the one hand to determine how there are altered the areas of occupational performance in persons by social phobia. In addition, one wants to establish also functions of the Occupational Therapist in the persons' treatment with this disorder. It is a question of an research of qualitative type using an interview semi constructed for the compilation of information and across the statement of history of life, axes of analysis decide to understand the impact of the Social Phobia.

Key words: anxiety disorders, social phobia, occupational performance.

\section{INTRODUCCIÓN}

Los trastornos de ansiedad conforman uno de los grupos de mayor incidencia en la salud mental ${ }^{(1)}$. Estos desórdenes ocasionan deficiencias, discapacidad o minusvalía; desde el punto de vista de la ocupación, en quienes lo padecen, puede interferir con la rutina diaria, las actividades productivas y las actividades de tiempo libre. Además los trastornos de ansiedad tienen frecuente comorbilidad con trastornos del ánimo, especialmente con cuadros depresivos, con consumo de sustancias y con trastornos asociados a factores somáticos. Por el deterioro ocasionado en la calidad de vida, al presentar estos cuadros psiquiátricos, es necesario un abordaje integral en la atención de salud mental ${ }^{(2)}$.

En Chile, dentro de los egresos hospitalarios por causa psiquiátrica en mayores de 15 años, los trastornos ansiosos conforman el segundo grupo de mayor egreso ${ }^{(3)}$, con un $25 \%$ del total de éstos, considerando en conjunto los trastornos ansiosos y depresivos, para el año 1993, de acuerdo con el Ministerio de Salud. Otros datos afirman que los trastornos de ansiedad, constituyen alrededor de un $50 \%$ de todas las consultas de psiquiatría ${ }^{(4)}$. Como nos indican estas cifras, los trastornos ansiosos forman un grupo importante.

Entre los trastornos ansiosos, se encuentra, la fobia social, definida por el DSM-IV, como "el miedo acusado y persistente a situaciones sociales o actuaciones en público por temor a que resulten embarazosas", lo cual provoca una respuesta ansiosa constante de tal magnitud que genera severas limitaciones en la vida del paciente. Respecto a esta patología no existen investigaciones en Chile que mencionen la intervención de Terapia Ocupacional. Estos antecedentes, motivaron a las investigadoras a diseñar, un estudio exploratorio que pudiera contribuir a definir la alteración en las áreas de desempeño ocupacional en estos pacientes y el rol del Terapeuta Ocupacional dentro de la intervención del equipo de salud mental en pacientes con fobia social.

\section{MARCO CONCEPTUAL}

La competencia social es un nivel general de eficiencia en el área de las relaciones interpersonales ${ }^{(5)}$, es altamente deseable y contribuye al estado de salud mental del individuo y de aquellos con quienes entra en contacto. Si los comportamientos y el grado de habilidad social resultan desadaptativos, las consecuencias pueden llevar a manifestar problemas de salud mental (6)

A través del proceso de socialización el ser humano llega a ser una personalidad social, una unidad que responde y es modificable dentro de un complejo de relaciones sociales. Además, la estructura de la personalidad humana constituye en gran medida un producto de la interacción social, que tiende a decaer cuando esta interacción cesa ${ }^{(7)}$. La evitación de la interacción social y 
la carencia de relaciones interpersonales, exacerbadas en el fóbico social, impiden que éste desarrolle menos aún sus habilidades sociales en un contexto real, lo cual, al largo plazo, generaría una retroalimentación negativa que favorece el déficit social.

Es evidente que las personas con fobia social, difícilmente podrán adquirir las habilidades necesarias para obtener un apoyo social satisfactorio, específicamente aquellas como competencia relacional, sociabilidad, bajos niveles de ansiedad social, habilidad social y estilo de afrontamiento que permita desarrollar recursos sociales ${ }^{(8)}$.

El autor, K. Davis, en su libro, La Sociedad Humana, explica la configuración social de la enfermedad mental que afecta a quien la padece de tal modo que la persona se vuelve impredecible para el resto, ya que la vida social se basa en la predecibilidad mutua de la conducta de unos con respecto a los otros. Lo anterior, se basa en la comunicación: por medio de ésta, las personas comparten lo que existe en sus respectivas mentes. Se espera que una persona note, emocional e intelectualmente, que ciertas normas rigen para ella, en su situación. Si no lo hace, su conducta tiende a volverse impredecible, la comunicación se desmorona y se le juzga como mentalmente desequilibrada.

Para ser considerado mentalmente perturbado, según refiere el autor, se debe diferir en forma sistemática y persistente de las suposiciones normativas y los hábitos mentales de los demás, que éstos ya no puedan entender la motivación y, por lo tanto, ya no sepan qué esperar de esa persona. En tales circunstancias la comunicación se vuelve imposible por su carácter de impredictibilidad. La persona perturbada no es capaz de ser influida por la comunicación con otros. Existiría un momento en que la comunicación ya no es posible, por ende, la persona se mantiene socialmente aislada en un sentido importante.

Con tal explicación de los trastornos mentales, desde un punto de vista social, podemos atribuir a la fobia social, las características de impredictibilidad y quiebre en la interacción social, secundarias al déficit de habilidades sociales y de competencia social, por supuesto, no concordante con las expectativas de los otros, con un posterior aislamiento que es vivido de forma egodistónica por parte del paciente.

La fobia social es un trastorno que se presenta con ansiedad clínicamente significativa como respuesta a un temor excesivo e irracional que aparece en ciertas situaciones sociales o actuaciones en público, lo que suele dar lugar a comportamientos de evitación de las situaciones temidas. La prevalencia global para la fobia social, según el DSM-IV, es entre 3 y 13\%.

De acuerdo al DSM IV ${ }^{(9)}$, la característica esencial de este trastorno es el miedo acusado y persistente a situaciones sociales o actuaciones en público por temor a que resulten embarazosas (Criterio A). La exposición a estos estímulos produce casi invariablemente una respuesta inmediata de ansiedad (Criterio B). Dicha respuesta puede tomar la forma de una crisis de angustia situacional o más o menos relacionada con la situación determinada. Aunque los adolescentes y adultos que padecen el trastorno reconocen que este temor resulta excesivo o irracional (Criterio C), puede que esto no suceda en los niños. En la mayoría de las ocasiones las situaciones sociales o actuaciones en público acaban convirtiéndose en motivo de evitación, si bien otras veces el individuo puede soportarlas aunque experimentando sumo terror (Criterio D). El diagnóstico puede efectuarse sólo en los casos en los que el comportamiento de evitación, el temor o la ansiedad de anticipación interfieren marcadamente con la rutina diaria del individuo, sus relaciones laborales y su vida social, o bien genera un malestar clínicamente significativo (Criterio E). En los menores de 18 años los síntomas deben haber persistido como mínimo durante seis meses antes de poder diagnosticar una fobia social (Criterio F). El miedo o los comportamientos de evitación no se deben a los efectos fisiológicos directos de una sustancia o enfermedad médica y no pueden explicarse mejor por la presencia de otro trastorno mental 
(trastorno de angustia con o sin agorafobia, trastorno por ansiedad de separación, trastorno dismórfico corporal, trastorno generalizado del desarrollo o trastorno esquizoide de la personalidad) (Criterio G). Si existe otro trastorno mental (por ejemplo, tartamudez, enfermedad de parkinson, anorexia nerviosa), el temor o el comportamiento de evitación no se limitan a la preocupación por su posible impacto social (Criterio H).

\section{METODOLOGÍA}

La metodología utilizada fue de tipo cualitativo y consistió en la aplicación de una entrevista semiestructurada a 3 usuarios de la red de atención Metropolitana Oriente de salud mental, 2 mujeres y un hombre. Todos ellos con diagnóstico de fobia social, asisten a recibir atención profesional en forma ambulatoria. El grupo se encuentran en la etapa de adulto joven de su desarrollo vital y corresponden a familias de estrato económico medio.

Las sesiones de entrevistas fueron individuales y se centraron en se narraciones de historias de vida $^{10}$. La determinación de códigos significativos en los relatos construidos, se agruparon en dimensiones que determinaron los ejes de análisis ${ }^{11}$. Los datos obtenidos se clasificaron en dos tablas con categorías determinadas.

Primer eje de análisis: Aspectos influyentes en el desarrollo de la Fobia Social / aspectos no vinculados al desarrollo de la Fobia Social.

Segundo eje de análisis: Áreas de desempeño Ocupacional afectadas como consecuencia de una fobia social / Áreas de desempeño ocupacional no afectadas

La muestra para esta investigación constó de tres personas con diagnóstico de fobia social, seleccionados según los siguientes criterios de inclusión: 1) Personas con diagnóstico de Fobia Social Específica, 2) Personas con diagnóstico de Fobia Social Generalizada, 3) Personas que aprueben ser parte de este estudio, por medio de la firma de un consentimiento informado ${ }^{12}$, 4) Personas que hayan desempeñado algún tipo de actividad productiva.

Para el primer eje categorial las dimensiones de análisis son las instancias de relaciones interpersonales con: familiares, pares, personas del sexo opuesto, figuras de autoridad y los factores que se asocian a estas relaciones: Hitos sociales negativos, evitación, compensación, autoimagen, autocontrol, roles intereses y valores.

Para el segundo eje categorial las dimensiones de análisis son las instancias dadas en la cotidianeidad de las personas es decir, en las actividades de la vida diaria, productivas y de juego y esparcimiento, donde se presentan las reales limitaciones que provoca la Fobia Social.

Las personas participantes que se comprometen en este estudio asisten a tres servicios de salud mental estatales, se contacta a los terapeutas ocupacionales de estos centros quienes actúan como intermediarios para acercarse a invitar a las personas.

La participación no representa riesgos para los colaboradores y se presenta como un beneficio la atención y orientación terapéutica que se ofrecen representados en los análisis de conflicto y entrenamiento del autocontrol.

Las entrevistas se desarrollan en un promedio de cuatro sesiones por personas en un ambiente preparado en el centro. Las narraciones, en el relato de las historia de vida se van guiando de acuerdo a las etapas del ciclo de vida y entorno al desarrollo de las actividades cotidianas.

En las entrevistas transcritas se identifican los códigos que se van relacionando con las dimensiones para los ejes de análisis. 


\section{RESULTADOS}

Las categorías de la Tabla $N^{\circ} 1$ se refieren a aspectos sociales y psicológicos que podrían verse afectados. Estos aspectos son considerados primarios para una posible alteración de las áreas de desempeño ocupacional. Las categorías de la Tabla $N^{\circ} 2$ corresponden a las tres áreas de desempeño ocupacional, de las cuales se analizarán los puntos que más podrían afectarse a causa de una fobia social. 
Tabla No1: Categoría “Aspectos influyentes en el desarrollo de la Fobia Social”

\begin{tabular}{|c|c|c|c|}
\hline \multirow{2}{*}{ Dimensiones } & \multicolumn{3}{|l|}{ Entrevistados } \\
\hline & $\mathbf{N}^{\circ} \mathbf{1}$ & $\mathbf{N}^{\circ} 2$ & $\mathbf{N}^{\circ} 3$ \\
\hline $\begin{array}{l}\text { Relaciones con } \\
\text { familiares }\end{array}$ & $\begin{array}{l}\text { Padre ausente. Madre Maltratadota } \\
\text { (relación de ambivalencia). Resentimiento } \\
\text { hacia hermana menor. }\end{array}$ & $\begin{array}{l}\text { Padre ausente. Mejor relación con la } \\
\text { madre. Estilo de crianza aprehensivo. }\end{array}$ & $\begin{array}{l}\text { Madre sobreprotectora. Padre exigente } \\
\text { y autoritario. }\end{array}$ \\
\hline $\begin{array}{l}\text { Relaciones con } \\
\text { pares }\end{array}$ & $\begin{array}{l}\text { Sin amigos. Sin sentido de pertenencia a } \\
\text { grupos. }\end{array}$ & $\begin{array}{l}\text { Pocos amigos en el colegio (vecinos } \\
\text { del barrio). Se excluía de juegos con } \\
\text { pares del mismo sexo. Evitaba y } \\
\text { rechazaba a compañeros nuevos. } \\
\end{array}$ & $\begin{array}{l}\text { Infancia solitaria. Influenciable } \\
\text { excesivamente por sus pares; buscando } \\
\text { aceptación comete actos indebidos. }\end{array}$ \\
\hline $\begin{array}{l}\text { Relaciones con } \\
\text { personas del sexo } \\
\text { opuesto }\end{array}$ & $\begin{array}{l}\text { Dificultades para relacionarse con sexo } \\
\text { opuesto (excepto en familia nuclear), evita } \\
\text { tal contacto. }\end{array}$ & $\begin{array}{l}\text { Dificultades para relacionarse con el } \\
\text { sexo opuesto. Pareja actual, en quien } \\
\text { se refugia y apoya. }\end{array}$ & $\begin{array}{l}\text { Dificultades para relacionarse con el } \\
\text { sexo opuesto, aunque logra tener } \\
\text { pareja. }\end{array}$ \\
\hline $\begin{array}{l}\text { Relaciones con } \\
\text { figuras de } \\
\text { autoridad }\end{array}$ & $\begin{array}{l}\text { Dificultades, manifestando síntomas } \\
\text { vegetativos de ansiedad. }\end{array}$ & $\begin{array}{l}\text { Temor excesivo al rechazo de figuras } \\
\text { de autoridad y personas poco } \\
\text { conocidas. }\end{array}$ & $\begin{array}{l}\text { Dificultades, manifestando síntomas de } \\
\text { ansiedad. }\end{array}$ \\
\hline $\begin{array}{l}\text { Hitos sociales } \\
\text { negativos }\end{array}$ & $\begin{array}{l}\text { Burlas de pares en enseñanza media, } \\
\text { frente al curso, experimentó síntomas } \\
\text { agudos y abandonó el colegio por tal } \\
\text { motivo. }\end{array}$ & $\begin{array}{l}\text { Burlas de compañero, en enseñanza } \\
\text { media (16 años), en presentación frente } \\
\text { al curso. Fue incapaz de continuar. }\end{array}$ & $\begin{array}{l}\text { Viaje a España, entre los } 9 \text { y } 11 \text { años, } \\
\text { se aislaba socialmente. }\end{array}$ \\
\hline $\begin{array}{l}\text { Comportamiento } \\
\text { ante situaciones } \\
\text { sociales : } \\
\text { Compensación } \\
\end{array}$ & $\begin{array}{l}\text { Refiere haber consumido sustancias } \\
\text { buscando desinhibirse socialmente. }\end{array}$ & $\begin{array}{l}\text { Refiere consumo de alcohol para } \\
\text { desinhibirse. }\end{array}$ & $\begin{array}{l}\text { Refiere consumo de alcohol en la } \\
\text { adolescencia para relacionarse con } \\
\text { sexo opuesto y olvidar problemas. }\end{array}$ \\
\hline $\begin{array}{l}\text { Comportamiento } \\
\text { ante situaciones } \\
\text { sociales : } \\
\text { Evitación }\end{array}$ & $\begin{array}{l}\text { Evita situaciones sociales. En eventos } \\
\text { sociales se aísla y presenta síntomas } \\
\text { vegetativos de la fobia social. } \\
\text { Las conductas de evitación la han llevado } \\
\text { al abandono escolar y no asistir a fiestas. }\end{array}$ & $\begin{array}{l}\text { Evita salir de la casa, incluso } \\
\text { acompañado. En eventos sociales se } \\
\text { aísla. Al hablar en público presenta } \\
\text { síntomas de fobia social y huye de la } \\
\text { situación. }\end{array}$ & $\begin{array}{l}\text { Evita situaciones con personas } \\
\text { desconocidas. En situaciones sociales } \\
\text { creencias de que va a hacer el ridículo. } \\
\text { Al hablar en público, presenta síntomas } \\
\text { vegetativos de la fobia social. }\end{array}$ \\
\hline Autoimagen & Pobre concepto de sí. Baja autoestima. & Autoimagen negativa. & Baja autoestima. \\
\hline Autocontrol & $\begin{array}{l}\text { En situaciones percibidas como } \\
\text { estresantes aumenta nivel de ansiedad y } \\
\text { huye. Sensación de descontrol de } \\
\text { síntomas. }\end{array}$ & $\begin{array}{l}\text { Incapaz de controlar creencias } \\
\text { irracionales y síntomas, huyendo de } \\
\text { situaciones percibidas como } \\
\text { estresantes. }\end{array}$ & Incapaz de controlar sintomatología. \\
\hline
\end{tabular}




\begin{tabular}{|l|l|l|l|}
\hline \multirow{2}{*}{ Roles } & $\begin{array}{l}\text { Roles de hija y trabajadora alterados, } \\
\text { siendo desempeñados } \\
\text { insatisfactoriamente. Nunca ha } \\
\text { experimentado el rol de amiga. } \\
\text { Rol de trabajadora no desempeñado según } \\
\text { intereses y motivación intrínseca. }\end{array}$ & $\begin{array}{l}\text { Rol de hijo alterado. } \\
\text { Rol de pareja alterado, muestra } \\
\text { dependencia afectiva. }\end{array}$ & $\begin{array}{l}\text { Todos los roles alterados. En rol de } \\
\text { hija no cumple con expectativas de los } \\
\text { padres. En rol de estudiante presenta } \\
\text { problemas conductuales. Rol de pareja } \\
\text { alterado (dependencia afectiva). }\end{array}$ \\
\hline Intereses & $\begin{array}{l}\text { Interés por apariencia física. } \\
\text { Proyecta retomar estudios, sin realizar } \\
\text { acciones para concretar. }\end{array}$ & $\begin{array}{l}\text { Actividades individuales o con pareja: } \\
\text { leer, andar en bicicleta, salir con pareja } \\
\text { y reunirse con amigos (acompañado } \\
\text { por su pareja). } \\
\text { No se pesquisa información al alifiesta interés por la apariencia } \\
\text { respecto. }\end{array}$ & $\begin{array}{l}\text { Mbandona actividades interesantes, } \\
\text { febido a la fobia social. }\end{array}$ \\
\hline Valores & $\begin{array}{l}\text { Valora bienes materiales y la belleza } \\
\text { física. } \\
\text { Valores alterados en el pasado (consumo } \\
\text { de sustancias). }\end{array}$ & $\begin{array}{l}\text { Valores alterados en el pasado } \\
\text { (consumo de sustancias). }\end{array}$ \\
\hline
\end{tabular}

Tabla N²: Categoría “Áreas de desempeño Ocupacional afectadas como consecuencia de una fobia social”

\begin{tabular}{|c|c|c|c|}
\hline \multirow{2}{*}{$\begin{array}{c}\text { Áreas de } \\
\text { Desempeño } \\
\text { ( Dimensiones) }\end{array}$} & \multicolumn{3}{|c|}{ Entrevistados } \\
\hline & $\mathbf{N}^{\circ} \mathbf{1}$ & $\mathbf{N}^{\circ} 2$ & $N^{\circ} 3$ \\
\hline \multicolumn{4}{|l|}{$\begin{array}{l}\text { ACTIVIDADES DE } \\
\text { LA VIDA DIARIA } \\
\text { (AVD) }\end{array}$} \\
\hline Socialización & $\begin{array}{l}\text { Se define como solitaria, a pesar de no } \\
\text { desearlo. }\end{array}$ & $\begin{array}{l}\text { La familia incentivaba la participación } \\
\text { en actividades y a tener amigos; el } \\
\text { paciente no realizaba lo aconsejado. }\end{array}$ & $\begin{array}{l}\text { Lograba socializar sólo con ingesta de } \\
\text { alcohol. }\end{array}$ \\
\hline $\begin{array}{l}\text { Comunicación } \\
\text { funcional }\end{array}$ & No se pesquisan alteraciones. & $\begin{array}{l}\text { Presenta alteraciones: miedo de } \\
\text { contestar el teléfono. }\end{array}$ & No se pesquisan alteraciones. \\
\hline Movilidad funcional & $\begin{array}{l}\text { En etapa aguda, aislada en casa, por } \\
\text { vergüenza a viajar en transporte } \\
\text { público. }\end{array}$ & No se pesquisan alteraciones. & Temor a viajar en transporte público. \\
\hline Expresión sexual & $\begin{array}{l}\text { Incapaz de comunicar sentimientos. } \\
\text { Nunca ha tenido pareja. }\end{array}$ & $\begin{array}{l}\text { Incapaz de coquetear, por miedo a ser } \\
\text { rechazado no intenta acercamiento. Ha } \\
\text { tenido parejas. }\end{array}$ & $\begin{array}{l}\text { Dificultad para relacionarse con sexo } \\
\text { opuesto. Ha tenido parejas. }\end{array}$ \\
\hline $\begin{array}{l}\text { ACTIVIDADES } \\
\text { PRODUCTIVAS }\end{array}$ & & & \\
\hline
\end{tabular}




\begin{tabular}{|c|c|c|c|}
\hline $\begin{array}{l}\text { Manejo del hogar } \\
\text { - hacer compras } \\
\text { - maneio de dinero }\end{array}$ & $\begin{array}{l}\text { No realizaba compras. Actualmente lo } \\
\text { hace. }\end{array}$ & $\begin{array}{l}\text { No realizaba compras por miedo a } \\
\text { interactuar con los vendedores. }\end{array}$ & No se pesquisan alteraciones \\
\hline Actividades educativas & $\begin{array}{l}\text { En enseñanza media, se agudizan los } \\
\text { síntomas, por lo cual abandona el } \\
\text { colegio. }\end{array}$ & $\begin{array}{l}\text { Abandonó universidad por } \\
\text { desmotivación. Presentaba problemas } \\
\text { para exponer en público, deseando } \\
\text { huir y deteniendo presentación. } \\
\text { Actualmente no ha tenido que } \\
\text { someterse a ésta experiencia. } \\
\end{array}$ & $\begin{array}{l}\text { En universidad, problemas para } \\
\text { exponer en público por manifestación } \\
\text { de síntomas. Dificultades para } \\
\text { interactuar con profesores. } \\
\text { Actualmente no está estudiando y no } \\
\text { sabe cuando retomará esta actividad. }\end{array}$ \\
\hline $\begin{array}{l}\text { Actividades } \\
\text { vocacionales } \\
\text { - adquisición del } \\
\text { empleo } \\
\text { - desempeño en el } \\
\text { empleo }\end{array}$ & $\begin{array}{l}\text { Incapaz de conseguir empleo, por } \\
\text { temor a interactuar. Empleos } \\
\text { gestionados por familiares. } \\
\text { No establece relaciones interpersonales } \\
\text { en el empleo. } \\
\text { No almuerza en el casino de } \\
\text { trabajadores por temor a ser vista } \\
\text { comiendo. }\end{array}$ & $\begin{array}{l}\text { Capaz de conseguir práctica laboral, } \\
\text { aunque con dificultad. } \\
\text { Logra interactuar en su empleo, } \\
\text { conversar y recolectar información con } \\
\text { los obreros, aunque el trabajo que } \\
\text { realiza es principalmente } \\
\text { administrativo. } \\
\text { No participa en actividades con } \\
\text { compañeros de trabajo, argumentando } \\
\text { falta de tiempo. }\end{array}$ & $\begin{array}{l}\text { Capaz de conseguir empleo. } \\
\text { El desempeño laboral no fue óptimo, } \\
\text { cometía errores por síntomas de } \\
\text { ansiedad. }\end{array}$ \\
\hline \multicolumn{4}{|c|}{$\begin{array}{l}\text { ACTIVIDADES } \\
\text { DE JUEGO Y ESPARCIMIENTO }\end{array}$} \\
\hline Explorar el juego & $\begin{array}{l}\text { No realiza actividades de } \\
\text { esparcimiento; es incapaz de identificar } \\
\text { intereses. }\end{array}$ & $\begin{array}{l}\text { Explora intereses centrados en } \\
\text { actividades solitarias. }\end{array}$ & $\begin{array}{l}\text { Abandonó actividades de interés. } \\
\text { Actualmente no realiza actividades de } \\
\text { esparcimiento. }\end{array}$ \\
\hline $\begin{array}{l}\text { Desempeño } \\
\text { en el juego }\end{array}$ & No desempeña tales actividades. & $\begin{array}{l}\text { Actividades como leer, andar en } \\
\text { bicicleta, salir con pareja y reunirse } \\
\text { con amigos. }\end{array}$ & $\begin{array}{l}\text { Evita reuniones sociales con } \\
\text { desconocidos. Asiste cuando hay } \\
\text { conocidos, disfruta la actividad, } \\
\text { aunque persisten deseos de abandonar } \\
\text { la situación. }\end{array}$ \\
\hline
\end{tabular}




\section{ANÁLISIS DE DATOS}

A continuación se detallarán y profundizarán las categorías en que fueron clasificados los datos entregados por los entrevistados:

\section{Relaciones Sociales}

Las familias de los pacientes estudiados se encuentran en las etapas del ciclo vital de: familia con hijos adolescentes y de plataforma de lanzamiento de los hijos del ciclo vital. Todas ellas han sufrido crisis no normativas como: intento de suicidio de un hijo, abuso de sustancias, abandono escolar, problemas de salud discapacitantes en padres, pérdida del trabajo, infidelidad del padre y abandono de la pareja. Se observa una alteración de los roles endofamiliares de los pacientes estudiados, de forma secundaria a la sintomatología fóbica; éstos se encuentran asumiendo funciones que no corresponden a su rol.

En las relaciones con los familiares es común el conflicto, tanto en los sistemas parento-filial como filial.

En el caso de la relación con los padres, con una figura paterna ausente y/o excesivamente exigente y una figura materna con conductas de sobreprotección, los entrevistados no cumplían con las expectativas de rol de los padres, siendo incluso maltratados psicológica y físicamente. Se constató la existencia de sentimientos ambivalentes de los hijos hacia los padres, quienes refieren no necesitarlos y expresan rencor por el abandono (afectivo o de hecho) sufrido en la infancia. Respecto al sistema filial, los pacientes estudiados, fueron comparados en desmedro de sus hermanos en la infancia y adolescencia e incluso sufrieron los efectos de las coaliciones en su contra. Se manifiesta una relación de inferioridad ${ }^{(13)}$, descrita por Eriksson, secundaria a la visión paterna, de esta forma, el hermano se siente con derecho a criticar y a traspasar los límites, generando rencor en el paciente. Las comparaciones entre los hijos verbalizando las expectativas de rol no cumplidas y las agresiones sufridas por los pacientes, son factores que incidieron en la génesis de la fobia social.

En cuanto a las relaciones con los pares pudimos observar que presentan tres características: superficialidad, complicidad y dependencia.

Las relaciones son definidas como superficiales, porque los entrevistados no desarrollaron confianza como para expresar- temores ni problemas, por ende, se asume que no existe una relación de confianza con pares. La forma de comportarse con los pares es imponiendo barreras, mostrando hostilidad o aparentando (sin mostrarse tal cual son), para evitar sentirse afectados por la crítica. Además, la baja autoestima de los pacientes, generada en gran parte en el núcleo familiar, los llevaría a creer que sus problemas - e incluso ellos mismos - son poco importantes.

Otra característica de las relaciones con los pares era la complicidad, donde éstos fueron un medio para desinhibirse y enfrentar la situación social, por medio de la ingesta de sustancias psicoactivas. Esto le ayudaría a interactuar en el grupo de amigos, desenvolviéndose de forma más cómoda y siendo socialmente aceptado. Los pacientes se relacionarían con sus pares en forma de dependencia afectiva.

Respecto a la relación con personas del sexo opuesto, este acercamiento resulta difícil, sobretodo en fase de galanteo. Al lograr establecer una relación de pareja, se vuelven dependientes de ésta.

En cuanto a la relación con figuras de autoridad, se establece con marcada inseguridad, siendo incapaces de realizar peticiones, dar a conocer sus ideas o exponer en situaciones formales porque se manifiestan la sintomatología. En esto, existiría de base un miedo irracional al rechazo, a la crítica o juicio. Además, las personas semejantes a las figuras paternas, generan en las personas sensaciones de miedo e incertidumbre. 


\section{Situaciones generadoras de ansiedad y formas de reacción ante situaciones sociales}

En los casos estudiados aparecen hitos sociales negativos, ocurridos en la niñez o adolescencia, situaciones en que han sido objeto de burla en público. Éstos se consideran factores ambientales influyentes en el desarrollo de la fobia social.

El comportamiento en situaciones sociales, se presentó con dos tipos de conductas: conductas de compensación y conductas de evitación. Las primeras otorgan protección en la situación social y serían: rodearse sólo de gente conocida, asistir a lugares conocidos, participando en actividades que no generen síntomas, o bien, asistir a lugares nuevos con compañía, afrontar la situación social consumiendo sustancias, para evitar aparición de sintomatología. Las conductas de evitación, como aislamiento y huida (abandonar el lugar o la situación) se presentan cuando no es posible realizar las conductas compensatorias y aparece la sintomatología. El grado máximo de la evitación observado, sería el abandono de la actividad por un periodo prolongado, como ocurrió en los tres casos, o incluso en forma definitiva.

Previamente a la situación social, los pacientes experimentan angustia anticipatoria; durante la situación, presentan ansiedad de exposición ${ }^{14}$, la cual genera una conducta de evitación, si el paciente pierde el control de la sintomatología, deseando huir de ese contexto o aislarse. Posterior a la evitación, sobrevienen sentimientos autodepreciatorios, de frustración y de culpa al evaluar su propio comportamiento. En esta fase, los pacientes reconocen sus temores como irracionales, aunque imposibles de controlar (vivencia egodistónica).

\section{Características de personalidad que condicionan la respuesta de ansiedad}

Otro punto común se refiere a la autoimagen, la cual es negativa, y se manifiesta en la baja autoestima. Respecto al autocontrol, se concluye que es pobre, pues los entrevistados presentan ante una situación considerada estresante, conductas primitivas y simples (regresión). No son capaces de enfrentar las situaciones de estrés, pues carecen de las habilidades necesarias para ello.

\section{Ocupación y Desempeño Ocupacional}

Respecto a los intereses, se observó que las entrevistadas, se enfocan en mejorar la apariencia física, y en cambio, el entrevistado manifestaba mayor interés en impresionar de manera intelectual.

En general las actividades que los entrevistados realizan son individuales en desmedro de aquellas colectivas, a pesar de desear participar en éstas, pero que no realizan, por su temor al rechazo social.

Los entrevistados presentan alteración en los roles ocupacionales adquiridos, en especial los productivos, porque debido a su sintomatología, abandonan tales actividades al ser incapaces de soportar la angustia generada al interactuar socialmente, con pares o superiores. En cuanto al rol de hijos, no cumplen con las expectativas de sus padres.

Por último, respecto a los valores, se pudo observar que los pacientes valoran la imagen proyectada a los demás: las mujeres en forma de apariencia física y el hombre en forma de inteligencia o capacidades. Se infiere que sus sistemas de valores se han visto alterados, en contra de lo que ellos mismos consideran correcto, al consumir sustancias o reunirse con personas que ejercían una influencia negativa, ambas conductas con el fin de lograr desinhibirse socialmente y evitar los síntomas. 


\section{Actividades de la Vida Diaria}

La primera Actividad de la Vida Diaria (AVD) analizada es la socialización. Para los entrevistados, la exposición a una situación social implica un sacrificio, aunque en general, el aislamiento tiene una connotación social negativa (castigo o sacrificio) ${ }^{(7)}$. Paradójicamente, la socialización era deseada por los entrevistados, aunque era reprimida por la sintomatología secundaria esta exposición (vivencia egodistónica). Para paliar tales efectos, los entrevistados consumían sustancias.

Los entrevistados tienen una socialización deficiente y es percibida como una característica negativa por el resto, que no es comprensible para los demás ${ }^{(7)}$. Se considera que la familia potencia este rasgo en la niñez, por una tendencia a la sobreprotección por alguno de los padres (en especial la madre), originando una incapacidad para confrontar los nuevos desafíos por parte del hijo.

Respecto a la comunicación funcional, uno de los entrevistados experimentaba tal grado de limitación como para no desear contestar el teléfono. Tal situación refleja la importancia, desde su apreciación distorsionada de la realidad, que los entrevistados otorgan a las expectativas del resto (locus de control externo).

En cuanto a la movilidad funcional, en dos de los casos analizados, se limitaba por el temor a usar transporte público, con creencias irracionales asociadas. Este factor disminuiría las oportunidades de socialización e induciría, de forma secundaria, a limitaciones como el abandono de actividades productivas o de tiempo libre.

Para finalizar, la expresión sexual, específicamente el galanteo. En los casos analizados, existían dificultades evidentes para relacionarse con el sexo opuesto, logrando sólo relaciones de amistad, en la mayoría de los intentos de búsqueda de pareja. Cuando se logra establecer una relación de pareja, los entrevistados tienden a utilizarla como un apoyo fundamental para establecer o mantener relaciones sociales.

\section{Actividades Productivas}

Esta es un área impactada negativamente; la dificultad o imposibilidad de realizar la actividad productiva, origina en los entrevistados sentimientos de frustración y baja autoestima, además de la evaluación negativa de su entorno social, al abandonar los roles de estudiante o trabajador.

Respecto al manejo del hogar (hacer compras y manejo de dinero), se ven alterados en uno de los entrevistados, incapaz de salir a comprar, por miedo a interactuar con los vendedores. Si bien las entrevistadas no presentaban mayores alteraciones en este ámbito, se ve interferido cuando las compras requieren uso de transporte público.

Los entrevistados presentaron problemas en las actividades educativas, que fueron desde la exclusión de actividades extraprogramáticas, pasando por dificultades para realizar de presentaciones en público, hasta abandonar la actividad educativa.

Por último, analizaremos las actividades vocacionales. En cuanto a la adquisición del empleo, se pudo observar dificultad e imposibilidad de conseguirlo, trabajando gracias a gestiones de los familiares.

Respecto al desempeño en el empleo, se observaron problemas en las relaciones interpersonales: son personas solitarias y con dificultades para socializar con pares. También se vio alterada la relación con los superiores, evidenciando una incapacidad para poder expresar asertivamente las necesidades. Se observó dificultad en la ejecución del trabajo cuando el éste requería interacción social frecuente. Por lo tanto, se puede concluir que existen empleos para los cuales los entrevistados simplemente no cumplen el perfil. 


\section{Actividades de Tiempo Libre}

Los entrevistados presentan graves trastornos en esta área, aunque éstos minimizan su importancia.

Se observa una restricción de los intereses de modo que las conductas se adaptan a las limitaciones y al tipo de personalidad, así los entrevistados ven disminuida la valoración de las actividades de tiempo libre, ya que éstas les reportan un feed-back negativo, tendiendo a cambiarlas por actividades que reporten mayor satisfacción y probabilidad de éxito, como actividades en solitario o con personas más cercanas.

Se concluye, entonces, que la motivación está perturbada. Al no existir motivación (ya que la actividad reporta displacer), no se conseguirá una exploración de las actividades que son propias según el rol y, por ende, se limita la variedad de intereses que la persona considera realizables, se evita todo tipo de actividad que requiera relaciones interpersonales, el rango de actividades se ve disminuido. Sin lugar a dudas, las actividades de tiempo libre que los entrevistados realizan en la adultez están afectadas por las posibilidades de exploración y ejecución que tuvieron durante su niñez y adolescencia.

De este modo, es posible vislumbrar que el desempeño ocupacional de estas personas se verá afectado en cuanto las actividades involucren relaciones interpersonales en menor o mayor grado.

\section{DISCUSIÓN}

\section{Observaciones acerca de las personas que padecen de fobia social}

El ser humano es considerado como un sistema abierto y su comportamiento ocupacional es el resultado de la interacción de este sistema ${ }^{(15)}$ con el medio ambiente.

En este análisis de la fobia social, podemos darnos cuenta de que su origen corresponde a factores genéticos, psicológicos y ambientales (socio-culturales), y que está mediado por la influencia familiar. Ésta, juega un papel determinante, por ser el ambiente social inmediato del individuo. Un punto importante respecto al ambiente son los hitos sociales negativos en periodos críticos (niñez y adolescencia) que influyen en la configuración de la personalidad, los cuales pueden desencadenar el desarrollo de periodos de agudización de las patologías psiquiátricas, en este caso la fobia social.

Los entrevistados presentan un evidente desequilibrio ocupacional, ocasionando trastornos que pueden llevar a estas personas a un aislamiento social patológico y discapacitante. En cuanto al impacto observado en las áreas de desempeño, podemos concluir que para los pacientes estudiados, la participación en actividades o tareas tanto de la vida diaria, como en trabajo y tiempo libre que requieran de una interacción con otras personas, sobretodo desconocidos, les ocasiona tal grado de ansiedad, que de no ser tratada puede llevar a un aislamiento total de la sociedad y provocar una disfunción ocupacional ${ }^{(15)}$, en la cual la persona no está satisfecha con su rutina. Este desequilibrio ocupacional, es el impacto final producto de varias disfunciones dadas en las distintas áreas de desempeño a través del tiempo, generando así una mala calidad de vida. La fobia social puede llegar a limitar tanto la vida de la persona, que ésta sea incapaz de realizar las AVD como hablar por teléfono o ir de compras, limitando también sus actividades productivas como el trabajo, la actividad académica y además sus actividades de tiempo libre, ya que en general, prefieren actividades de tipo solitario y cuando asisten a actividades sociales, como reuniones o fiestas, necesitan rodearse de personas conocidas o consumir psicotrópicos para desinhibirse.

Desde la perspectiva del Modelo de Ocupación Humana, se alterarían los tres subsistemas ${ }^{(15)}$. Primero, la alteración en la volición incluye la causalidad personal, los intereses y los valores. Esta alteración implica una disminución en la causalidad personal y en la efectividad sobre el medio ambiente, ambos presentes en nuestros entrevistados en especial en situaciones 
sociales; los intereses, se irían modificando y excluyendo la interacción social que genera la sintomatología (por ejemplo, coleccionar monedas, decorar la casa, estar con la familia); y por último, los valores, centrados en evitar conflictos con el resto, que refleja una marcada dependencia de la aprobación externa. Segundo, respecto a la habituación, referida a los hábitos, se afecta en el sentido de que los entrevistados adaptan la rutina a sus limitaciones, descartando toda actividad genere ansiedad. Los roles se alteran, cuando no cumplen las expectativas como hijos (hijo rebelde, hijo sobreprotegido), estudiantes (abandono escolar y evitación de situaciones académicas) y trabajadores (trabajos esporádicos). Probablemente también se encuentren alterados otros roles no detectados en esta investigación. Por último, la ejecución es deficiente cuando la ansiedad interfiere en una situación que implica interacción social, tanto en las actividades de la vida diaria, como ir de compras, en el trabajo o estudio y en las actividades de tiempo libre, en las cuales puede reaccionar con evitación y huida de la situación, o bien, si le es imposible huir, vive la situación con gran angustia.

Respecto a la socialización de las personas con fobia social, se puede decir que los medios tecnológicos actuales (chat, correo electrónico) facilitan la comunicación de éstas, por generar menos ansiedad que la interacción cara a cara o telefónica. Si bien estos medios pueden ser una ayuda para el paciente, sólo debieran ser tomados, como un apoyo temporal y no permanente para la socialización. Además, los entrevistados presentan un sentimientos de ambivalencia frente a la socialización, porque a pesar de temer las relaciones sociales, desean tenerlas, resultando menos angustiante evitarlas y racionalizando su incapacidad con ideas como: escaso interés, la prescindencia de entablar estas relaciones o argumentando obligaciones que no dan tiempo para actividades sociales. En este punto, el paciente se discapacita, ya que él mismo retroalimenta sus limitaciones, limitando su desempeño progresivamente, aunque con la ambivalencia y los mecanismos de defensa mencionados.

Inicialmente la persona intenta adaptarse al aislamiento, creando un entorno protector de la ansiedad (dependencia de sustancias, dependencia de personas significativas, y un estilo de vida socialmente evitativo), disminuyendo el posible impacto social negativo e incluso llegando al extremo de no tener desafíos sociales, eliminados efectivamente. Se puede plantear que una persona con fobia social no es capaz de desarrollar una fortaleza yoica funcional, y sufre porque desearía ser y actuar de otra forma, es decir, vive egodistónicamente su problema de salud. En algún momento su rutina se vuelve insatisfactoria, debido a la incapacidad de concretar sus intereses, a su escaso apoyo social o a no poder llevar una vida social como el resto de las personas, y, entonces, puede llegar a presentar comorbilidad con episodios depresivos, trastorno de ansiedad generalizada o abuso de sustancias, utilizadas para enfrentar las situaciones sociales sin ansiedad. Esto explica que el paciente consulte por trastornos comórbidos asociados la fobia social ${ }^{(16,13)}$ y no por ésta propiamente tal.

Cuando estas personas consultan se considera necesario y urgente intervenir con un equipo multidisciplinario. Dentro de éste la intervención psicosocial que le compete a la Terapia Ocupacional, se fundamenta en la existencia de factores modificables, de tipo psicológico, ambiental y ocupacional.

\section{Aporte de la Terapia Ocupacional en el tratamiento de la fobia social}

Como se mencionó anteriormente, desde el punto de vista ocupacional la persona con fobia social tiende a un desequilibrio que lo puede llevar a limitaciones severas, por lo cual se cree que la Terapia Ocupacional puede aportar en el tratamiento de esta enfermedad.

En la intervención, el Terapeuta Ocupacional actúa como modelador de nuevas conductas y estrategias útiles para que el paciente pueda integrarse al mundo social, recobrando la motivación a explorar y ejecutar actividades desafiantes, basándose en una causalidad personal y sentido de eficacia mejorados, y a aumentar las actividades que incluyan socialización. ${ }^{17}$ 
En relación al tratamiento oportuno, la intervención de Terapia Ocupacional estaría basada el entrenamiento de habilidades sociales y de manejo de la ansiedad, para favorecer el desempeño ocupacional, mediando la exposición a desafíos ocupacionales, de forma progresiva, que le permitan desarrollar tales habilidades y enfrentar exitosamente eventos similares en el futuro. ${ }^{18}$ Se puede incluir también elementos para mejorar la autoestima. En estas aproximaciones se incluirán elementos del Modelo de Ocupación Humana y de los enfoques conductuales, dando énfasis en la ejecución de ocupaciones del individuo y posteriormente analizar sus atribuciones frente al éxito y al fracaso, para luego confrontarlas y cambiarlas, aplicando este aprendizaje en un nuevo enfrentamiento a los desafíos ocupacionales. Estas medidas, con intervenciones farmacológicas de base, permitirían controlar los síntomas vegetativos que provocan la ansiedad en situaciones sociales, inicialmente por medios externos (incluyendo mayor apoyo del Terapeuta Ocupacional) y posteriormente, con el aprendizaje del propio paciente.

Otro punto del tratamiento, se refiere a la orientación vocacional, en la que el Terapeuta Ocupacional puede dar una visión objetiva entre capacidades y exigencias de las preferencias ocupacionales del paciente. En ocasiones las personas con fobia social no cumplen con el perfil para ciertos tipos de trabajo de su interés, con lo cual deben abordar con dos líneas: mejorar capacidades individuales del paciente para enfrentar esta situación, mencionadas en el párrafo anterior, y buscar desafíos ocupacionales más apropiados a sus habilidades. Como se constató en los resultados, estos pacientes muchas veces pierden la objetividad y buscan actividades productivas discordantes con sus habilidades.

Sin embargo, un aspecto fundamental es la modificación del estilo de vida, o rediseño ocupacional, desde edades tempranas, lo cual supone un diagnóstico precoz y tratamiento oportuno de la fobia social o por lo menos prevención primaria en grupos de riesgo (niños excesivamente tímidos o con hipersensibilidad social) ${ }^{(19)}$. Esta área de intervención, considera la desensibilización progresiva en las ocupaciones realizables por el niño o adolescente en riesgo, según su nivel de evolución, favoreciendo un desempeño ocupacional normalizante que incluya actividades propias según roles, edad, contexto, etc. El tratamiento debe ser ideado individualmente, con desafíos progresivamente mayores y ejecutado en conjunto con la familia, con el fin de que ésta apoye el tratamiento.

A futuro, sería posible plantear un proyecto gubernamental para mejorar la calidad de vida de los niños en riesgo de presentar fobia social, que incluyera tanto al sistema de salud como al sistema educacional, realizando un tamizaje en edades claves (por ejemplo, entrada al colegio e inicio de la adolescencia) en consultorios, policlínicos y establecimientos educacionales. Además, con esto se ampliarían las funciones del Terapeuta Ocupacional en el sistema educacional, haciendo detección precoz de niños hipersensibles sociales. Este tamizaje debiera ser considerado para la intervención propiamente tal y las orientaciones vocacionales realizadas en las escuelas.

De este modo, en el nivel primario, se intervendría con promoción y prevención, por medio de la creación de políticas que incluyan talleres de habilidades sociales en el sistema educacional dentro de la malla curricular, las cuales se impartirían a todos los niños sin distinción y también a nivel comunitario como por ejemplo en los COSAM, juntas de vecinos, clubes sociales, etc.

En el nivel secundario, al igual que en el nivel anterior, se requiere de un trabajo conjunto con los sistemas educacionales, consultorios e incluso empresas para realizar un diagnóstico precoz y así orientar las acciones para un tratamiento oportuno ejecutado por un equipo multidisciplinario.

Por último, en el nivel terciario, la rehabilitación psicosocial y la reinserción comunitaria, la intervención estaría orientada a reestablecer el equilibrio ocupacional que ha perdido el 
fóbico social. Este aspecto fue abordado en nuestra propuesta de intervención a la familia de los casos estudiados.

\section{REFERENCIAS BIBLIOGRÁFICAS}

${ }^{1}$ CARRIÓN O., BUSTAMANTE, G. Ataques De Pánico Y Trastornos De Fobia Y Ansiedad. Segunda edición. Buenos Aires, Argentina. Editorial Galerna. 2003. p.19.

2 Plan Nacional De Salud Mental y Psiquiatría [en línea].Chile, Ministerio de Salud, 2001 [fecha de consulta: 26 de Junio de 2004]. Disponible desde Internet: $<$ http://www.minsal.cl/ici/s_1/u_14/Cap4Red.pdf>

3 Las enfermedades mentales en Chile: Magnitud y Consecuencias [en línea]. Chile. Ministerio de Salud, 1999 [fecha de consulta: 16 Marzo 2006]. Disponible desde Internet:< http://www.minsal.cl/ici/s_1/u_14/CAPITULO\%20II.PDF>

${ }^{4}$ FLORENZANO R. Trastornos Ansiosos. En: Psiquiatría. Santiago de Chile. Editorial Mediterráneo, 1999. p. 105.

5 ARÓN A. M., MILICIC N., Vivir con otros. Programa de Desarrollo De Habilidades Sociales. Segunda Edición. Santiago de Chile. Editorial Universitaria. 1994. p.17

${ }^{6}$ POPE, A., McHALE, S., CRAIGHEAD, W. Mejora de la Autoestima: Técnicas para niños y adolescentes. Barcelona. Editorial Martínez Roca.1996. p.111

${ }^{7}$ DAVIS, K., La Sociedad Humana.Tercera Edición. Buenos Aires. Editorial Universitaria de Buenos Aires. 1969.

${ }^{8}$ NTP 439: El apoyo social [en línea]. España: Ministerio de Trabajo y de Asuntos Sociales, Instituto Nacional de Seguridad e Higiene en el Trabajo, Centro Nacional De Condiciones De Trabajo. [fecha de consulta: 01 de Octubre 2004]. Disponible desde Internet: < http://www.mtas.es/insht/information/index.htm >

${ }^{9}$ AMERICAN Psychiatric Association. DSM-IV: Manual Diagnóstico y Estadístico de los Trastornos Mentales. Barcelona. Editorial Masson. 2001.

${ }^{10}$ LOLAS, F. La medicina como narrativa. Revista Médica de Chile, 1992; 120, 810 -815.

${ }^{11}$ COURTES, J. Introducción a la semiótica narrativa y discursiva. Buenos Aires: Hachette 1980

${ }^{12}$ RUEDA, L. Consideraciones éticas en las investigaciones con seres humanos. Revista Chilena de Terapia Ocupacional 2003. ISSN 0717 - 6767 No 4 p. 19 - 24

RUEDA, L. Ética e investigación en seres humanos en: bio Etica: fundamentos y dimensión práctica. Mediterráneo, Santiago. 2004

${ }^{13}$ PAPALIA, D., WENDKOS, S. y DUSKIN, R. Desarrollo Humano. 4a EDICIÒN Bogotá. Editorial McGraw-Hill, 2001, p.32. 
${ }^{14}$ LOLAS, F. La perspectiva psicosomática en medicina. Editorial Universitaria, Santiago 1995

${ }^{15}$ HOPKINS, H., SMITH, H. Willard \& Spackman, Terapia Ocupacional. Octava Edición. Madrid. Editorial Panamericana, 2001, pp. 47, 77-78.

${ }^{16}$ TIMIDEZ y fobia social aumentan el consumo de alcohol y tranquilizantes. La Tercera. Santiago, Chile, 13 de Agosto de 2005, p. 39, col.1.

${ }^{17}$ CLARK, F. Reflections on the human as an occupational being: Biological need, tempo and temporality. Journal of Occupational Science, 1997. 4 (3) 86 - 92

${ }^{18}$ CLARK, F. (1993). Occupation embedded in a real life: Interweaving occupational science and occupational therapy. American Journal of Occupational Therapy, 47,1993. p 1067-1078.

${ }^{19}$ SIMPOSIO Actualización en Trastorno por Ansiedad Social o Fobia Social (2003, Santiago, Chile), Centro de Convenciones Laboratorio Chile. Presentaciones del Dr. Jorge Mahaluf: Diagnóstico diferencial y estrategias farmacológicas. Diagnóstico Clínico. 